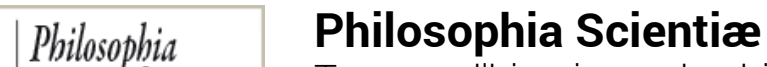

Scientie Travaux d'histoire et de philosophie des sciences

$15-3 \mid 2011$

L'espace et le temps

\title{
Penser ensemble le temps et l'espace
}

\section{Bernard Guy}

\section{OpenEdition}

Journals

Édition électronique

URL : http://journals.openedition.org/philosophiascientiae/684

DOI : 10.4000/philosophiascientiae.684

ISSN : 1775-4283

Éditeur

Éditions Kimé

Édition imprimée

Date de publication : 1 octobre 2011

Pagination : $91-113$

ISBN : 978-2-84174-569-2

ISSN : $1281-2463$

Référence électronique

Bernard Guy, « Penser ensemble le temps et l'espace », Philosophia Scientiæ [En ligne], 15-3 | 2011, mis en ligne le 01 octobre 2014, consulté le 10 décembre 2020. URL : http://journals.openedition.org/ philosophiascientiae/684; DOI : https://doi.org/10.4000/philosophiascientiae.684 


\title{
Penser ensemble le temps et l'espace
}

\author{
Bernard Guy \\ École nationale supérieure des mines de Saint-Étienne - \\ PRES Université de Lyon (France)
}

Résumé : Nous proposons de penser ensemble les concepts d'espace et de temps : ils concernent les mêmes degrés de liberté des éléments du monde et fonctionnent toujours en tandem. Leurs fondements doivent être discutés, non dans une pensée de la substance (chacun est défini par une série de caractères qui lui sont propres), mais dans une pensée de la relation (chacun se définit en opposition à l'autre). Nous opposons des relations spatiales à des relations temporelles, ou encore des relations d'immobilité à des relations de mobilité relative. La décision de la frontière entre ces deux ensembles de relations est sujette à arbitraire : nous avons une grande flexibilité dans les définitions associées des paramètres d'espace et de temps; elle ne fait pas non plus l'économie de difficultés conceptuelles ou logiques semblables à celles rencontrées dans la mécanique quantique. Il faut revoir dans cette perspective autant le concept de temps que celui d'espace : le temps ne coule pas, il est changement de relation, il est mouvement; l'espace est abstrait à partir de relations constantes ou morceaux constants de mouvement. Les mouvements relatifs qui expriment ces relations, changeantes ou non, contiennent toujours un aspect spatial et un aspect temporel, comme pile et face de la même réalité. Nous proposons de voir plus généralement dans toute relation un aspect spatial (l'écart qui sépare les deux termes de la relation) et un aspect temporel (le parcours du chemin qui les relie). Sur cette base, nous proposons un programme de recherche pour reprendre un certain nombre de problèmes fondamentaux de la physique contemporaine, ainsi que des pistes pour reprendre ce que nous disons du temps et de l'espace dans les sciences humaines et sociales, la culture, et jusque dans la vie quotidienne.

Abstract: We propose to think together the concepts of space and time: they involve the same degrees of freedom of the elements of the world and always work in tandem. Their foundations are to be discussed, not inside a thinking of substance (each is defined by a series of characters of its own) but inside a thinking of relation (each is defined in opposition to the other). We oppose spatial relations to temporal relations, or relative immobility to relative mobility relations. The decision of the boundary between the two is subject to 
arbitrary (we have a great flexibility in the definitions of the associated space and time parameters) and does not avoid logical and conceptual difficulties similar to those encountered in quantum mechanics. We should revise from this perspective both concepts of time and space: time does not flow, it is a change in the relations, it is movement; space is abstracted from constant relations or constant portions of movement. The relative movements that express these (changing or not changing) relations always include a spatial aspect and a temporal aspect, as heads and tails of the same reality. We propose to see more generally in any relation, a spatial aspect (the gap between the two terms of the relation) and a temporal aspect (the travel along the path that connects them). On this basis, we propose a research program to examine a number of fundamental problems of contemporary physics, as well as ideas to rethink about the way we use the words related to time and to space in human and social sciences, culture and even everyday life.

\section{Introduction}

Dans ce travail, nous voulons discuter les concepts d'espace et de temps, non selon une pensée de la substance, comme on le fait le plus souvent, mais selon une pensée de la relation, et chacun des deux concepts étant pensé en relation avec l'autre. Comprenant qu'espace et temps fonctionnent toujours en tandem, nous sommes conduits à revoir autant la signification du temps que celle de l'espace. Signification du temps : il ne coule pas, nulle part, il n'est la propriété d'aucun point, il est relation, il est changement de relation entre les points matériels dans l'espace, il est mouvement. Signification de l'espace : il s'appuie sur des relations supposées invariables entre ses points constitutifs, exprimées par des fractions constantes de mouvements ou de déplacements, renvoyant au temps qui les permettent. Si la réflexion sur le concept de temps occupe ici, comme souvent, plus de place, elle concerne bien l'espace : il faut également le revisiter dans son association avec le temps. Ces diverses réflexions nous conduisent à faire des propositions de recherche autant en physique qu'en ce qui concerne notre compréhension plus quotidienne du temps et de l'espace. Dans le présent article, la pensée de la relation est discutée comme élément indispensable du contexte de l'argumentation; dans la mesure où nous proposons une généralisation (toute relation contient à la fois un aspect temporel et un aspect spatial), elle est aussi un élément central de notre étude.

L'article est découpé comme suit. Dans une première partie (section 2), nous décrivons succinctement les deux modes de pensée (substance/ relation). Dans une deuxième partie (section 3 ), nous opposons des relations appelées spatiales entre les éléments du monde (sur lesquelles nous construisons l'espace) à des relations appelées temporelles (sur lesquelles nous construisons le temps). Nous nous arrêtons ensuite (section 4) sur les caractéristiques d'une 
telle pensée de la relation ou, plus précisément ici, pensée du mouvement, qui font apparaître un certain nombre de limites conceptuelles et logiques (situation d'incomplétude, présence d'incertitudes, de boucles de récursivité, de contradictions). Puis (section 5), c'est l'occasion de faire apparaître, lors de notre discussion sur espace et temps, les étapes ou conditions de la pensée de la relation, en une structure abstraite très générale. Nous poursuivons par deux parties d'application :

- à la physique tout d'abord (section 6), en évoquant quelques questions qui peuvent être relues dans la présente vision;

- à la culture de façon plus large ensuite (section 7), en reprenant certaines apories du temps et la solution que nous en proposons.

Nous terminons par quelques lignes de conclusion à propos de la question que pose le titre de cet article (section 8) : pouvons-nous penser « ensemble » l'espace et le temps? La bibliographie présentée est nécessairement limitée, sur un ensemble de sujets extrêmement vaste.

\section{Sur deux modes de pensée}

Comment pensons-nous le monde, comment le disons-nous? Nous nous comportons le plus souvent comme si nous pouvions le regarder de l'extérieur, et mettre en correspondance les éléments que nous y distinguons avec les mots d'un « espace » de connaissances, indépendant et au-dessus de lui, tel un ciel d'idées platoniciennes, un monde d'essences éternelles. Ainsi pour penser ou dire le « cheval», nous le mettons spontanément en correspondance avec une définition du cheval (animal ayant tels caractères - les caractères possibles sont comme donnés à l'avance - ), ainsi que nous le faisons dans un dictionnaire, quitte à compléter la première définition par d'autres. Il n'est pas alors nécessaire d'opposer les éléments du monde les uns aux autres, de les comparer, chacun est vu en lui-même, pour lui-même. Pour en revenir à notre exemple, nous n'avons pas besoin de penser « en même temps » à la vache ou au cochon pour dire que le cheval n'en est pas. Nous qualifions cette pensée de pensée de la, ou en termes de, substance. Les caractères de cette pensée ont été exposés, avec des variantes, par de très nombreux auteurs. Nous pouvons citer, pour leur rôle décisif, Platon, Aristote [Aristote 1997] ou, plus près de nous, Descartes [Descartes 1644], Newton [Newton 1687], Kant [Kant 1781].

Cette pensée, qui s'appuie sur une apparente nature ou substance propre des éléments du monde, montre ses limites, spécialement aujourd'hui. Une autre pensée s'est développée au fil des siècles, en parallèle au courant dominant. Elle a été énoncée d'abord par Héraclite [Héraclite 1964] et les présocratiques. En sautant à la période actuelle et sans chercher l'exhaustivité ni les nuances, on peut en trouver des éléments de synthèse chez des chercheurs comme Edgar Morin [Morin 1986], [Morin 2005]. Des auteurs contemporains tel Jullien (en particulier à propos de la pensée chinoise, voir par exemple 
[Jullien 1993], [Jullien 2006]) et Bitbol [Bitbol 2010] en ont repris l'analyse et l'exposition. Ce second mode de penser insiste sur le fait que nous ne sommes pas à l'extérieur du monde, mais en son sein : nous ne voyons que des relations ou des oppositions entre les éléments du monde. Pour penser au cheval, nous faisons (ou avons fait) en réalité une série de comparaisons entre les différents animaux; nous énumérons ce qui rend le cheval différent d'eux. Dire ce qu'est le cheval revient en somme à faire la liste de tous les animaux qu'il n'est pas. Cela implique que, d'une certaine façon, en pensant au cheval, nous pensons en même temps à tous les autres animaux, vivants et passés (et au-delà aux corps inanimés... ) et faisons des partitions, des séparations en leur sein pour le définir. C'est d'ailleurs ainsi que la pensée scientifique procède aujourd'hui de façon plus rigoureuse pour situer le cheval dans un système de classes selon une approche phylogénétique (combien de caractères le séparent de tel ou tel animal ?) par opposition à la démarche antérieure de construction d'un arbre d'évolution (a-t-il en propre tel ou tel caractère?). Nous qualifions cette pensée de pensée de, ou par, la relation, ou de pensée en termes de relations. Dans le passé, divers auteurs (par exemple, [Montaigne 1580], [Pascal 1670]) ont insisté, sinon sur la relation, du moins sur la nécessité de construire notre connaissance en dualités, voire contradictions, et d'allier les contraires.

Même si cette intelligence de la relation est insuffisamment mise en valeur dans la pensée contemporaine, nous estimons que c'est véritablement ainsi que nous pensons ou commençons à penser, y compris, et nous en venons au propos de cet article, pour des catégories fondamentales comme l'espace et le temps. Ainsi, dans la conception première que nous venons de rappeler, nous imaginons que nous mettons en correspondance avec l'espace et le temps (supposés découverts par l'expérience) une série de qualificatifs permettant d'en rendre compte de façon indépendante l'un de l'autre. Il n'est pas alors besoin de «penser » à l'espace pour parler du temps, ou de «penser» au temps pour parler de l'espace.

Dans le présent texte, nous voulons développer le point de vue que les concepts de temps et d'espace doivent être discutés dans une pensée de la relation et, précisément, en relation l'un avec l'autre : pour penser le temps nous avons besoin de l'opposer à l'espace, pour penser l'espace nous avons besoin de l'opposer au temps. L'espace et le temps sont pensés ensemble, en opposition l'un à l'autre. Ce sera une façon de reconnaître que, si nous associons si souvent espace et temps pour décrire le cadre des phénomènes offerts à notre investigation, ce n'est pas du tout un hasard : ce sont pile et face de la même pièce, ce sont les deux visages d'une même réalité. En parlant ainsi de l'espace et du temps, nous verrons aussi comment fonctionne la pensée de la relation. Parmi les auteurs scientifiques, nombreux ont discuté les concepts d'espace et de temps en termes de relation (voir par exemple [Mach 1904], [Whitehead 1922], [Barbour 1982], [Earman 1990], [Rössler 1998], [Assis 1999]), mais en envisageant chaque concept de son côté, sans voir, comme nous le faisons ici, 
que ce sont les mêmes relations qui sont en jeu pour les deux, et les couplent donc de façon indissociable.

\section{Relations spatiales, relations temporelles}

Abordons maintenant la construction des concepts d'espace et de temps dans une pensée de la relation. Notre monde nous est donné une seule fois et non trois, comme les présentations habituelles semblent le montrer en les poussant à la limite (une fois avec l'espace seul sans le temps, une fois avec le temps seul sans l'espace, une troisième avec espace et temps) : il n'y a pas d'étiquette sur ce qui est temps ni étiquette sur ce qui est espace. Dans notre compréhension, l'espace et le temps n'ont pas de réalité propre, ce sont des concepts abstraits à partir de ce que nous pouvons dénommer les relations entre les éléments du monde. Nous répartirons ces relations entre des relations baptisées spatiales, et des relations baptisées temporelles (on notera que, en parlant de pensée de la relation, nous utilisons ce dernier mot au second degré, par rapport à ce que nous appelons au premier degré aussi relations, spatiales et temporelles ; le contexte d'utilisation des mots évitera les ambiguïtés, et nous ne discuterons pas les problèmes de récursivité qui se posent à cet endroit). Comment pouvons-nous comprendre l'opposition, ou la séparation, entre les relations spatiales et les relations temporelles? Nous avons analysé cela dans de nombreux travaux, voir par exemple [Guy 2004], que nous pouvons reprendre brièvement. Plusieurs expositions sont possibles. Partons d'une approche pragmatique et regardons comment nous construisons effectivement, depuis des millénaires, d'une part les règles et repères d'espace qui nous servent pour jalonner nos territoires, et d'autre part les horloges et repères temporels qui nous servent pour ordonner les événements. Pour l'espace, nous plantons des bornes dans notre terre, ou nous désignons des règles, et, à l'aide des triangulations de la géométrie, établissons des cartes. Pour le temps, nous repérons les différentes positions du Soleil (dans son mouvement relatif par rapport à la Terre), ou les différentes positions d'un repère associé à un système mécanique plus ou moins sophistiqué, ou par extension nous regardons les positions d'un photon dans une boîte que nous appelons horloge atomique et par rapport aux parois desquelles il se déplace; nous mettons en correspondance les événements de notre histoire avec ces positions. Ce faisant, parmi les multiples points que nous pourrions déclarer mobiles, nous en privilégions un sur lequel nous pouvons nous mettre d'accord (selon des modalités qui peuvent être discutées).

Nous considérons que, par ces opérations, nous fonctionnons déjà, peutêtre sans nous en rendre compte, selon un régime de la pensée par relation ou pensée par opposition. Nous faisons en effet une partition dans notre monde entre un ensemble de points de positions relatives stables, immobiles ou invariantes (les bornes ancrées sur les montagnes; les extrémités de la règle) et un 
point, ou un ensemble de points, mobile(s) par rapport aux premiers : le soleil, les aiguilles de l'horloge, les grains de sable dans le sablier, les photons, etc. (nous pourrions rajouter les vagues de la mer, les nuages... ). C'est bien grâce à cette opposition que nous définissons à la fois les uns et les autres. Ce faisant, nous n'exprimons rien de « positif » sur chacun des deux groupes de points en particulier. Nous exprimons simplement un point de vue relatif : nous « immobilisons » les premiers par rapport aux seconds déclarés mobiles (et plus précisément, déclarés mobiles à vitesse constante dans une horloge), sans être sûr fondamentalement de la réelle immobilité des uns (ou, ce qui est équivalent, de la constante mobilité des autres), en fonction de mots qui seraient donnés extérieurement au monde, prononcés par l'usage de critères indépendants de lui (des règles et des horloges). Il faudrait dire plutôt en bref que les premiers points ne bougent « pas trop» par rapport aux seconds. Dans cette construction par opposition, il y a un degré d'arbitraire, comme des expériences simples de pensée nous le montrent [Guy 2004]. En effet, si nous vivions beaucoup plus longtemps, beaucoup plus lentement, les montagnes (sur lesquelles nous avons planté nos bornes) bougeraient comme les vagues de la mer. Nous pourrions nous en servir pour définir le temps. Ce qui servait donc à définir l'espace servirait à définir le temps. Mais nous pouvons aussi imaginer : si nous vivions moins longtemps, à un rythme plus rapide, les grains de sable dans le sablier ne bougeraient pas durant notre vie entière. Nous pourrions nous en servir pour repérer l'espace. Ce qui servait donc à définir le temps servirait à définir l'espace... Certains diront : « C'est une belle allégorie», mais à un moment donné, nous allons pouvoir nous arrêter quelque part et annoncer : «Voilà l'espace, voilà le temps ». Eh bien non! Dans ce continuum de déplacements relatifs, l'on ne peut pas s'arrêter de façon absolue, de façon définitive; il n'y a pas de coupure préexistante, il n'y a pas d'outils en dehors du monde qui pourraient nous donner accès à de l'espace et du temps fondamentalement distincts l'un de l'autre. Ce n'est pas un problème de perception subjective qui pourrait être résolu par des appareils plus fins : ni l'observateur, ni les outils de mesure, qui sont tous à l'intérieur du monde, ne peuvent s'arrêter à une frontière absolue. La constante mobilité et l'immobilité sont pensées ensemble; nous sommes dans une situation d'autoréférence, conduisant à des propositions indécidables. Nous faisons une coupure «provisoire » entre espace et temps, en arrêtant une régression qui nous mènerait à l'infini. Nous accédons alors à une description plus ou moins précise du monde. C'est ce qui est exprimé dans les transformations de Lorentz de la théorie de la relativité. Et dans cette coupure se joue notre liberté de choix, nous pouvons parler de « choix arbitraires » (laissés à notre libre arbitre). Nous construisons aujourd'hui une physique 《appuyée »sur la lumière, dans la décision $c=c s t e$ ( $c$ est la vitesse de la lumière). Mais nous pourrions construire une physique où c'est la vitesse du mouvement apparent du soleil autour de la terre qui est constante, comme on l'a fait dans le passé (ou plus précisément la vitesse de rotation sidérale), ou d'autres physiques encore. Et nous pourrions contempler de l'extérieur ces 
physiques contradictoires entre elles, rendues chacune cohérente (même si telle ou telle physique serait plus compliquée à mettre en œuvre).

\section{Pensée du mouvement et de la relation}

En résumé de ce qui précède, espace et temps sont construits en opposition l'un à l'autre dans une collection de mouvements relatifs, associés aux mêmes degrés de liberté des éléments du monde : l'espace (ou les relations spatiales) est (sont) construit(es) sur des mouvements «arrêtés », ou infiniment lents, par rapport à d'autres mouvements qui nous permettent de construire le temps (les relations temporelles). Ce sont les deux faces d'une même pièce. La dualité temps/espace ne vient pas d'une dualité de substances, mais de la multiplicité des éléments du monde et du possible partage de leurs relations en deux (ou plusieurs) groupes. Ce faisant nous donnons au mouvement un caractère primaire, permettant à la fois de parler d'espace (amplitude du mouvement), et de temps (procès du mouvement). Et nous associons à toute portion de réalité sensible un ou des mouvements. Si divers auteurs ont insisté sur les caractères spécifiques du mouvement (par exemple [Chatelet 1993], [Maldiney 2001], [Webb 2006]), ils n'ont pas été appelés comme nous à un ré-examen des significations associées des concepts de temps et d'espace (voir [Guy 2004, 2010a]). La révision concerne l'espace tout autant que le temps; et le mouvement lui-même que nous dissocions de sa vitesse (la vitesse résulte de la comparaison de deux mouvements).

La conception de l'espace est en effet à reprendre en relation avec le mouvement. L'espace a son sens dans la connexion des différents points qui le constituent, ce qui fait apparaître le rôle des déplacements. Quel sens aurait-il comme collection de points sans lien les uns avec les autres? La position d'un point renvoie à un déplacement depuis une origine. Le lien entre l'espace et le mouvement (le déplacement), et à travers eux le temps, nous conduit du côté de la biologie et des sciences cognitives où l'on étudie les modalités de l'appréhension de l'espace par le mouvement ([Alegria 1983], [Imbert 1983], [Moraïs 1983], [Ninio 1983], [Berthoz 1997]). Dans ses travaux, ce dernier auteur souligne que, de façon générale, la perception, et, à travers elle, la connaissance, sont intimement liées à l'action et au mouvement. Il résume cela en disant : la perception est une action simulée. Le cerveau n'est pas un organe représentationnel, mais un simulateur biologique : «Les propriétés les plus raffinées de la pensée et de la sensibilité sont des processus dynamiques. »Dans ce contexte, la construction de l'espace dans le cerveau est liée à l'exploration du monde par le sujet, et ce lien met pratiquement en œuvre des capteurs sensoriels associés aux muscles, à l'oreille interne et à tout le corps. L'ensemble de ces capteurs définit ce qu'Alain Berthoz appelle «le sens du mouvement». On peut dire que l'espace tel que nous le construisons dans notre cerveau est une simulation interne du mouvement; il n'est pas codé de façon explicite comme 
« espace » mais se présente comme le nœud de l'ensemble des trajectoires qui s'y inscrivent. Poincaré [Poincaré 1902] lui-même avait vu ce lien entre espace et mouvement. Cet auteur posait la question de départ de savoir pourquoi nous avons besoin de construire un espace géométrique : nous le faisons dans la mesure où un tel espace nous est commode pour représenter nos déplacements et les modalités de notre action. Nous ne nous représentons pas les corps dans l'espace géométrique mais nous raisonnons sur les corps comme s'ils étaient situés dans un espace géométrique. La géométrie est la description des phénomènes que nous appelons déplacements, c'est-à-dire des changements externes, qui sont compensés par des changements internes de notre corps mesurables par des capteurs vestibulaires. Cette géométrie est basée sur les corps solides : s'il n'y en avait pas dans la nature, souligne Poincaré, il n'y aurait pas de géométrie. En reliant les travaux de Berthoz à ceux de Poincaré, nous mettons en évidence une chaîne allant de l'action à l'espace géométrique, via les représentations cérébrales. Pour Teissier [Teissier 2009], la droite réelle mathématique (au sens des nombres réels) ne peut être séparée de sa construction dans la cognition : nous imaginons que nous avançons le long d'elle à vitesse constante; elle est ainsi paramétrée de façon cachée par le temps. En revenant à notre propos, nous dirons que l'espace, comme simulation interne du mouvement, est lié de façon indissociable au temps, qui est lui-même une autre face de ce mouvement, en comprenant le mouvement de façon très générale, au-delà de celui du sujet qui l'a le premier expérimenté. C'est le mouvement au sens où nous disons que la géométrie est la science des déplacements. Cette révision de la compréhension de l'espace pourra mériter de nouveaux approfondissements.

Avec celle de l'espace, il faut revoir aussi la signification du temps : comme nous l'avons dit, le temps ne coule pas, nulle part, il n'est la propriété d'aucun point, il est relation, il est changement de relation dans l'espace, il est mouvement (on repense aux travaux de Piaget [Piaget 1967] sur la construction de la notion de temps chez l'enfant).

Nous nous interdirons dorénavant de parler du temps tout seul, sans parler en même temps de l'espace, et réciproquement, nous nous interdirons de parler de l'espace tout seul, sans parler en même temps du temps.

\section{Caractéristiques de la pensée de la relation}

Mais dire : «le temps est défini par le mouvement »(l'espace est défini par le mouvement), mérite discussion. Nous sommes en effet confrontés à une contradiction : le temps serait défini à la fois avant et après le mouvement? (L'espace serait défini à la fois avant et après le mouvement?) Comment définir le mouvement, sans avoir d'abord défini l'espace ainsi que le temps lui-même? Ce type de question nous conduit à la frontière de la logique et nous ouvre à des réflexions plus générales. Nous trouvons à propos de la théorie de la relativité une structure intellectuelle comparable à celle de la mécanique quantique, et 
abondamment commentée à son endroit ; elle n'avait pas été vue à propos de la discussion des concepts de temps et d'espace. Cette structure est caractérisée par les propriétés suivantes, que nous avons déjà évoquées ici pour certaines [Guy 2010a] :

- contenu d'incertitude : nous ne sommes pas sûr de la stricte correspondance entre les mots et les choses; nous ne sommes pas sûr des valeurs numériques que nous attribuons aux grandeurs physiques. Ce qui se traduit ici par : nous ne sommes pas complètement sûr du statut de mobilité ou d'immobilité de tel point matériel, nous ne sommes pas sûr de la valeur numérique attribuée à sa vitesse (nous pouvons d'ailleurs associer à cette situation des relations mathématiques d' « incertitude », voir [Guy 2004]);

- situation d'incomplétude : le discours construit ne dit pas tout; il ne se suffit pas à lui-même, on y observe la

- présence de choix arbitraires, c'est-à-dire soumis au libre arbitre et non strictement imposés par la réalité ; leur origine est extérieure au discours de départ (situation d'incomplétude; voir aussi [Léonhardt 2008]) : nous choisissons de dire que la lumière a une vitesse constante et nous assumons ensuite ce choix dans la mise en œuvre de nos mesures et dans le fonctionnement de nos équations. Ces choix peuvent conduire à la

- possibilité de modèles de pensée contradictoires entre eux : nous pouvons construire plusieurs physiques fondées sur des choix différents du phénomène de vitesse décidée constante. Nous sommes ainsi confrontés à des

- situations de récursivité où les mots sont définis les uns à partir des autres, et dont nous ne pouvons sortir qu'en montrant, en nous contentant de montrer, quelque chose de la « réalité », sans être sûr de son adéquation à des mots qui seraient comme définis en dehors du monde. Nous le faisons en disant : ce phénomène (la propagation de la lumière) nous sert de base « constante».

\section{Construction de la pensée de la relation}

\section{Les étapes ou conditions de la pensée de la relation}

En mettant en œuvre, ainsi que nous l'avons fait, la construction des concepts d'espace et de temps apparaît une série de conditions sur lesquelles repose la pensée de la relation, comme autant d'étapes élémentaires de son édification. Nous pouvons y voir un ensemble d'opérations intellectuelles élémentaires ou une structure abstraite très générale pour penser le monde, au-delà des concepts de temps et d'espace ([Guy 2010b]; voir aussi [Dujardin 2009]).

- Existence d'une scène «vide ». La pensée de la relation suppose de façon implicite une scène vide où puisse se penser l'établissement ou la 
rupture des liens entre les éléments du monde. C'est la condition de la création de ces liens. Nous pourrions appeler « espace» ce vide, mais nous faisons une distinction entre le vide d'un côté et l'espace de l'autre, ce dernier appuyé sur les relations que nous avons appelées spatiales. Ce fond commun nous sert précisément à définir également les relations temporelles (et le temps) par opposition aux relations spatiales. Dans d'autres registres ce vide se relie à ce qui est appelé : l'indicible, le fond d'incomplétude, l'être, le réel voilé (par opposition aux représentations que nous construisons), etc. Ce vide est le fond duquel nous détachons les éléments que nous distinguons, ou voulons distinguer, dans le monde et dont nous allons étudier les relations (le vide est encore l'absence de relation).

- Opération de coupure. Sur ce fond, nous établissons des regroupements des éléments du monde en classes (les points immobiles dans les solides par exemple), en positionnant une frontière ou coupure les séparant d'une ou de plusieurs autres classes (les points mobiles). En somme, pour définir A, nous avons besoin (à la place d'un axiome d'identité) de dire « A est non non-A », en l'opposant à B, C, D, etc.

- Relation d'équivalence. Ce faisant, nous affirmons une certaine équivalence (cf. les relations d'équivalence en mathématiques) entre les points d'une classe. Le solide est vu comme un tout solidaire, on le pense dans son unité. Ainsi, pour la pensée de l'espace qui résulte d'un regard d'ensemble sur un groupe de points.

- Nécessité de poser un absolu (au moins provisoire). Cette pensée par relation montre une limite en ce qu'elle conduit à des régressions à l'infini. Comment décider qu'un point bouge ou non par rapport à un autre, comment décider qu'il mérite d'être associé ou non à d'autres points dans un «solide», de compter dans un ensemble de « relations spatiales » (c'est-à-dire invariables) ? C'est-à-dire, plus généralement, comment décider de la proximité ou non de tel élément par rapport à tel autre, pour décider de le mettre ou non d'un côté de la frontière du groupe? Pour juger de l'invariance des relations entre deux points, il faut s'assurer de la constance de la distance qui les sépare; il faut pour cela disposer d'un mouvement de vitesse constante dans le phénomène de référence. Mais comment juger de ce dernier si ce n'est en le comparant à un autre mouvement, et ainsi de suite. Pour arrêter ces régressions, pour stabiliser en somme cette pensée de la relation, nous avons besoin (nous ne pouvons faire autrement), d'attribuer un caractère absolu (invariant, archétypal), au moins provisoirement, à tel ou tel « phénomène » du monde que nous choisissons et désignons. Nous avons dit : ce phénomène, que nous vous montrons, se décrit par une propagation à la vitesse $c=c s t e$, par rapport à ces règles que nous décidons invariables. Nous assumons ce choix dans le bouclage de nos propositions et de nos mises en œuvre expérimentales, en attendant de faire un choix éventuellement plus adapté. C'est faire 
une sorte de retour à la pensée de la substance, mais qui est maintenant conscient de son caractère fragile, même s'il est indispensable pour penser. Pour le phénomène étalon, nous nous contentons de dire « $\mathrm{A}$ est $\mathrm{A}$ » sans dire « $\mathrm{A}$ n'est pas non-A ». Cette opération ne relève cependant pas de la pensée de la substance au sens habituel dans la mesure où elle procède tout autant par la désignation d'un phénomène particulier dans des circonstances particulières (« regardez ce que j'utilise comme étalon dans telle situation expérimentale »); ce n'est pas équivalent à l'attribution du caractère (absolu et général) choisi à une propriété substantielle de la nature. La condition de la symétrie de la relation entre deux éléments A et B demande aussi le postulat d'un absolu, commun aux deux termes de la relation. Derrière tout cela se pose la question de la nécessaire interprétation (choix de l'《absolu ») et de l'autorité nécessaire pour le proclamer (cf. les travaux de Ricœur [Ricœur 1969] sur l'herméneutique, dont il faut étendre le champ à la physique même). Les caractéristiques de la pensée de la relation (section 4) peuvent être comprises par les conditions discutées dans la présente section.

\section{L'espace et le temps dans toute relation}

Nous pouvons aller plus loin et généraliser les concepts d'espace et de temps, à travers le concept de relation, ou de mouvement. Chaque fois qu'il y a relation nous dirons qu'il y a espace. Chaque fois qu'il y a relation, nous dirons qu'il y a temps. Espace dans l'amplitude de la relation, dans l'écart qu'elle suppose. Temps dans le déploiement de la relation, dans le parcours du « trajet » ou du lien qui rejoint l'un à l'autre les deux termes qu'elle conjugue. De façon indissociable, toute relation à la fois sépare (espace) et relie (temps). Sans nous affranchir des difficultés conceptuelles et logiques dont nous avons parlé, c'est à la lumière de ces propositions qu'il faut relire divers problèmes, autant dans la culture, les sciences humaines et sociales que dans les sciences de la nature, et, en particulier, penser l'incorporation nécessaire d'une dimension temporelle dans les multiples concepts que nous construisons. Ainsi le temps de l'évolution biologique qui exprime le lien caché entre les différents animaux que nous classons par relations... Dans la pensée de la substance au contraire, il n'y a pas de temps et l'on met en regard de façon instantanée, sans se poser la question du temps, l'élément du monde au mot qui le désigne dans l' « espace» de la connaissance (ou le «logos»). Il faut reprendre dans cette perspective l'examen de la façon dont nous concevons, construisons et ordonnons nos connaissances. 


\section{Le temps dans la physique}

\section{Deux constats}

Revenons maintenant à la physique d'aujourd'hui pour laquelle nous pouvons faire deux constats. Dans le premier, observons que la question du temps reste mal résolue. On l'évite en prenant d'emblée (en plus et à côté des paramètres $x, y$ et $z$ ), un paramètre $t$, toute discussion sur sa construction étant d'avance écartée. Les nouvelles théories qui envisagent de nombreuses dimensions d'espace (les variantes de la théorie des cordes) ne se posent pas non plus généralement la question du temps. Dans le second constat, remarquons que de nombreuses voix s'élèvent pour parler de malaise en physique, ou pour montrer un certain nombre de difficultés dans les théories existantes (voir par exemple [Selleri 1998], [Smolin 2006]). Nous ne pouvons examiner ici tous les débats, tant la littérature sur le sujet est abondante. Aucune de ces voix pourtant ne conteste fondamentalement l'existence du temps et sa représentation actuelle dans la physique. Rapprochant ces deux constats, et à la suite des lignes qui précèdent, il nous semble qu'une réflexion approfondie, de nature conceptuelle, sur le temps et ses liens à l'espace, est un préalable indispensable à tout progrès en physique. Outre les difficultés pour lesquelles nous avons proposé une solution ou un début de solution [Guy 2004], la compréhension du temps et ses liens avec l'espace exposée à l'instant peut avoir une portée plus grande; elle permet de discuter de nouvelles questions en physique même. Evoquons rapidement quelques points.

\section{Le temps en mécanique quantique}

Certains auteurs, tels Rovelli [Rovelli 1990], [Rovelli 1991], montrent que l'on peut se passer du paramètre temps dans le formalisme de la mécanique quantique. D'autres soulignent la mauvaise coexistence entre le temps de la relativité et certaines expériences de la mécanique quantique, qui leur font douter de l'existence du temps «à une certaine échelle ». Ne peut-on pas éviter ces difficultés en remarquant que le temps n'existe à aucune échelle, et n'est qu'un simple marqueur? Il est à la limite absurde de vouloir attacher un «temps propre» à telle ou telle particule, alors que le temps est relation. En se libérant du concept de temps propre et en insistant sur le caractère conventionnel du marqueur que nous appelons temps, la question de l'échelle (microscopique ou macroscopique) à laquelle on pourrait ou non associer un paramètre temporel aux équations de la mécanique quantique ou de la relativité ne se pose plus. Le problème des jumeaux de Langevin s'éclaire de la même façon : la différence d'âge correspond à un point de vue différent par rapport à un marqueur mobile et non à un âge réel «propre » sans signification. Comprendre les relations de Lorentz comme relations d'incertitude [Guy 2004], peut aussi conduire à apaiser les relations entre relativité et mécanique 
quantique. Les incertitudes que nous voyons là portent sur la propriété de mobilité ou d'immobilité des repères matériels sur lesquels nous sommes obligés de nous appuyer pour repérer nos positions et nos temps. Elles peuvent s'exprimer par une «petite» vitesse inconnue marquant l'écart ou le déplacement entre deux repères géométriques sur lequel nous écrivons les transformations de Lorentz.

\section{La vitesse de propagation de la lumière}

D'autres questions se posent à propos de l'hypothèse de la constance de la vitesse de la lumière, dans le temps ou dans les différentes directions de l'espace (problème de l'isotropie de propagation, par exemple [Nodland \& Ralston 1997]), de l'existence ou non de vitesses supraluminiques, etc. On peut les aborder en remarquant que l'on est a priori devant des choix conventionnels, certes indispensables à poser, et à assumer après coup, mais que l'on ne peut écarter la possibilité ou la nécessité d'en faire d'autres suivant les situations rencontrées... Les lois de la nature ne sont pas strictement imposées par celle-ci. Nous avons une certaine latitude pour les écrire, et, dans le présent contexte, on comprendra que l'on regarde avec circonspection certaines théories physiques postulant des univers parallèles régis par d'autres lois, comme si ces dernières y étaient prescrites indépendamment de choix humains. Poincaré avait déjà mis en valeur le pluralisme théorique [Poincaré 1902], [Poincaré 1905] (voir aussi [Greenberg 1997], [Mizony 2010]) que nous retrouvons ici.

\section{L'irréversibilité du temps}

Sur la question de l'irréversibilité encore, nous pouvons dire : le premier problème du temps n'est pas son irréversibilité mais son existence. Le temps, pas plus que l'espace n'existe tout seul; temps et espace sont construits à partir du monde, ils sont relations. C'est parce que le temps est associé à un ou des mouvements dans l'espace (ce n'est pas un pur «flux » indépendant de l'espace), par opposition à des relations spatiales stables, que nous pouvons opposer son irréversibilité à la réversibilité des relations spatiales. L'opposition entre la réversibilité et l'irréversibilité est ainsi fondée sur cette opposition et simplement comprise comme la possibilité ou l'impossibilité de revenir en arrière pour certaines des particules du monde par rapport à d'autres qui marqueraient le repère d'espace pour ce retour potentiel : l'incertitude sur la définition des paramètres d'espace et de temps limite fondamentalement cette possibilité de parler d'un retour en arrière. La réversibilité et l'irréversibilité sont pensées ensemble, comme l'espace et le temps, et leur limite est sujette à approximation. Les lois mêmes de la mécanique sont réversibles dans la seule mesure où l'on peut définir un repère d'espace « fixe», ce qui est toujours sujet à approximation, le repère étant pratiquement appuyé sur des points matériels. 
À ce titre, on peut tout aussi bien parler de l'irréversibilité de l'espace (cf. [Guy 2004]); ou encore, de l'irréversibilité de l'espace-temps dans la façon de le comprendre dans le présent article. Cette irréversibilité de base, conceptuelle ou ontologique, se raccorde avec l'irréversibilité pratique déjà comprise pour des systèmes à grand nombre de particules, où l'on retrouve la flèche du temps en prenant en compte les effets des incertitudes sur les conditions initiales, les effets des perturbations de divers types sur les trajectoires (voir analyse dans [Guy 2008]).

\section{Le temps dans la culture}

Pour faire une transition vers le temps de la culture, remarquons que, de même que l'espace est multiple (multiplicité des lieux), nous pouvons concevoir une multitude de relations entre les éléments du monde et une multitude de temps, en portant notre attention sur telle ou telle partie du monde, sur tel ou tel mouvement ([Pomian 1984] parle aussi de cette pluralité). Mais, par delà cette multitude de temps, il nous a été indispensable de définir un temps unique pour communiquer entre nous et relier ces différents temps. Ce temps unique, c'est le temps de la physique (ou le temps habituel, on pourrait dire universel), dont nous avons parlé. Ce temps unique est un simple repère extérieur aux choses. Il ne signifie à la limite rien par rapport à la marche de telle ou telle partie du monde.

Comprendre cela nous donne un point de vue pour aborder les problèmes du temps qui continuent de se poser dans le domaine de la philosophie, des sciences humaines et sociales, et de toute la culture. À titre d'exemple, regardons certaines apories fameuses du temps (on aura compris qu'il faudrait parler tout autant des apories de l'espace, voir [Guy 2004]), en nous demandant après tant d'autres : comment le temps existe-t-il, lui qui est composé du passé, qui n'existe plus, du futur, qui n'existe pas encore, et du présent, néant coincé entre deux néants? L'association du temps et de l'espace donne des éléments de réponse [Guy 2010b]. Il faut pour cela revenir à la source : le «mouvement». Ainsi, comme nous l'avons dit, en nous intéressant à tel ou tel mouvement, nous opposerons ce qui est spatial (les mouvements «très lents ») à ce qui est temporel (les mouvements « sensibles »). Mais le temps ainsi défini reste «à côté » de l'espace (à côté dans son sens le plus banal). Un morceau de solide stable est en quelque sorte épargné par le temps qui coule «ailleurs ». Les catégories grammaticales du temps : passé/présent/futur concernent non pas cette partie temporelle mobile extérieure aux choses stables, ni le temps universel qui n'est qu'un repère, mais l'ensemble espace + temps dans la multiplicité des relations spatiales et temporelles. Après Einstein [Einstein 1905], nous aurions déjà pu relier les catégories du temps à l'association indivisible espace-temps. Ce raccord exprime que la langue concerne le fonctionnement 
du monde dans son ensemble et non tel ou tel morceau plus ou moins artificiellement séparé (le temps par rapport à l'espace). Ainsi quand nous parlons de présent, nous parlons à la fois du nuage qui se fait et se défait, renvoyant au temps du changement, et de la montagne immobile et présente depuis des millénaires, renvoyant à l'espace. Il est absurde de dire que cette montagne disparaît constamment dans le passé pour renaître dans le présent. Elle est provisoirement hors du temps. Nous pourrions parler de passé présent, pour indiquer cette partie spatiale (non purement temporelle) du temps grammatical. Et cette montagne est aussi un présent du futur (un futur présent, au moins futur proche). C'est tout le sens de sa présence. Ce qui est bien passé, passé du passé, c'est le moment qui a compté comme frontière de fluctuance (instant d' «agrégation »), où cette montagne a surgi, même si nous devons pour cela envisager de longues durées. De même pour le vrai du futur, le futur futur, qui verra ou non une désagrégation de la montagne et une nouvelle combinaison de ses éléments. Par l'adjonction de deux termes temporels pour désigner une même expérience (passé présent, futur futur, etc.) nous pouvons faire apparaître des nuances sur le caractère mixte des catégories grammaticales à la fois spatiales et temporelles (sur cette question du temps, de l'espace et de la langue, voir aussi [David 2004], [Asic 2004], [Hagège 2009]). Une autre façon de décrire les qualités du présent serait de chiffrer les proportions de ce qui change dans l'environnement du sujet, ou dans ce à quoi il prête attention : un présent à $90 \%$ spatial et $10 \%$ temporel pour indiquer que la proportion de ce qui change ou de ce que le sujet voit changer (ou décide de voir changer) autour de lui est de $10 \%$. On conçoit que l' ' épaisseur » de ce présent, sa durée, est fonction de ce pourcentage, en supposant implicitement qu'il ne peut pas varier trop rapidement : un présent à $90 \%$ temporel — c'est la tempête, le chaos - est plus fugitif qu'un présent à $10 \%$ temporel, comme l'immobilité du désert. Plusieurs définitions des catégories passé/ présent/ futur peuvent ainsi coexister, chacune étant reliée à une classe de mouvements ou de changements repérés dans l'environnement du sujet (il n'y a pas un temps unique qui imbiberait tout). On peut en garder plusieurs ou, au contraire, en privilégier une, celle par exemple basée sur tel phénomène physique permettant une large communication. Par comparaison, on «spatialise » alors ce qui n'est pas relié à des changements. Chaque sujet expérimente ainsi, là où il se trouve, une certaine répartition entre les différentes modalités de son «temps »; elles vont se traduire par des «proportions » entre ce qu'il attribuera (pour lui) aux catégories grammaticales passé/ présent/ futur. La connexion par l'effet de signaux et de déplacements de matière entre les lieux où sont positionnés les sujets, va relier de façon objective entre elles les « grandeurs » temporelles associées à chacun. On dispose ainsi d'une méthode, appuyée banalement sur la conservation de la matière, pour relier les temps des uns et des autres, et donner un contenu quasi quantitatif à des propositions langagières reliant l'écoulement du temps au jeu de l'espace : «le futur vient d'ailleurs », «le présent s'en va à côté », «le passé revient », etc. Si l'on ne porte pas son attention sur tel repère commun 
fondant la distinction sociale passé/ présent/ futur, on comprend que certains puissent dire : « no future».

Les difficultés qui se posent à propos du temps dans la culture (multiplicité des temps, sens des catégories passé/ présent/ futur) s'éclairent par ce double constat - de multiplicité des relations/ multiplicité des temps et - de choix nécessaire (inévitable) d'un temps unique. Telle est une solution que nous proposons pour les apories du temps, fondée sur une nouvelle compréhension du temps et des relations temps espace. Elle permet de réconcilier le temps des physiciens à celui des humanités. Elle demande un examen détaillé de la répartition de ce qui se passe autour du sujet parlant. On conçoit qu'une telle analyse rigoureuse soit lourde à mener et que les expressions de la vie de tous les jours ne puissent éviter des incohérences logiques.

\section{Conclusion : penser ensemble l'espace et le temps}

Penser ensemble l'espace et le temps? Oui, au moins de façon provisoire, dans l'image mentale qui relie l'un à l'autre dans le mouvement. Oui, un instant seulement, en un tiers inclus éphémère, avant que l'on ne « s'arrête ». Souvenons-nous en effet d'Aristote dans la Physique [Aristote 1961] : « il faut s'arrêter ». Seule cette halte permet le discours et la connaissance, et, après elle, nous ne supportons plus la contradiction et le tiers inclus. Mais ce que nous pouvons souligner maintenant c'est le caractère conventionnel, fragile, jamais définitif, de cet arrêt, au sein d'une transhumance infinie, qui sans cesse nous demande de remettre sur le métier nos formulations. Arrêt qui ne doit pas être compris non plus en opposition au mouvement, mais avec lui, comme condition de sa définition. La pensée chinoise ne nous invite-t-elle pas de son côté à une inséparation du dynamique et du statique, du dynamique et de la configuration?

Si nous avons utilisé le terme d'opposition, et des mots apparentés, comme ingrédient de la pensée de la relation (opposition entre des ensembles d'éléments, entre des qualités associées, etc.), nous devrions parler plutôt de composition : les différents ensembles ne sont jamais concevables les uns sans les autres. Ce terme exprime un lien plus «pacifique», plus «symbiotique », que celui porté par le mot d'opposition. Les relations elles-mêmes composent entre elles : la symétrie, l'équivalence, la réversibilité composent avec la dissymétrie, la différence, l'irréversibilité; le vide, l'absence de relation, composent avec la matière, la relation; l'extérieur compose avec l'intérieur de la frontière, la désignation de celle-ci avec sa traversée; le temps compose avec l'espace, etc. (voir [Dujardin \& Guy 2011]).

Cette étape du penser ensemble l'espace et le temps, malgré son caractère provisoire, est fondatrice. Elle nous permet de comprendre la genèse des mots 
et des concepts correspondants, leurs limites, et les difficultés qui y sont cachées, depuis la culture et la philosophie jusqu'à la physique. La théorie de la relativité est là, dans cette association du temps, de l'espace et du mouvement. L'identification que nous pouvons faire en physique entre temps et mouvement n'est pas sans conséquences concrètes. Elle nous conduit à utiliser trois coordonnées pour construire le temps, non en ajout des coordonnées spatiales, mais comme trois d'entre elles à adjoindre à celles des points de l'espace : elles correspondent à celles d'un point particulier, exprimées dans le même repère, et dont le mouvement nous sert pour définir le temps. Nous contemplons alors un espace-temps renouvelé, plus compact que celui de la relativité standard qui reliait les mesures d'espace et de temps sans unifier les concepts. Ceci a de multiples conséquences pour le fonctionnement du formalisme (écriture des lois générales de conservation en physique, des transformations de Lorentz, des équations de Maxwell ; reprise des équations de la gravitation... ) comme nous l'avons proposé de façon préliminaire dans [Guy 2010c] et [Guy 2010d]. De nombreux auteurs ont constaté le bon, ou meilleur, fonctionnement des équations de la physique en utilisant un paramètre temporel à trois dimensions (par exemple [Demers 1975], [Pappas 1978], [Pappas 1979], [Ziino 1979a], [Ziino 1979b], [Tsabary \& Censor 2004], [Chen 2005], [Franco 2006]). Mais ils n'en ont pas compris la véritable portée : le temps n'a pas trois dimensions! C'est un scalaire que nous construisons pour ordonner nos événements, mais nous le faisons à partir d'un mouvement qui, lui, est repéré par trois coordonnées, tout comme l'est un repère qui se déplace dans l'espace. Ce repère peut être associé à un objet céleste ou un photon dans une horloge atomique : dans ce cas l'orientation du dispositif de détection n'est pas quelconque et la valeur de l'étalon temporel incorpore de façon cachée les directions de l'espace. L'utilisation du paramètre temporel tri-dimensionnel est une façon technique de résoudre des problèmes techniques posés par la théorie de la relativité. Audelà d'une amélioration de cette théorie, c'est à un progrès conceptuel que nous prépare l'approche présentée ici. Pour aller vers la résolution de problèmes plus profonds dont nous avons évoqué certains, comme ceux reliant relativité et mécanique quantique. La clé de leur compréhension est la grande flexibilité que nous avons dans la définition du temps, de l'espace et des variables associées et l'assouplissement corrélatif de la contrainte de concaténation des processus physiques pour maintenir un temps universel.

En conclusion, le présent travail doit être considéré comme un angle d'attaque pour reprendre divers points qui touchent au temps et ses relations plus ou moins cachées avec l'espace. Il donne les premiers éléments d'un programme pour examiner de nombreuses questions qui se posent en physique fondamentale aujourd'hui. Les réflexions exposées touchent également à des aspects concrets et quotidiens de notre façon de comprendre l'espace et le temps et d'en parler. De ce côté, elles proposent aussi des pistes de recherche. 


\section{Remerciements}

L'auteur remercie tous ceux qui ont manifesté, de diverses façons, une connivence avec son entreprise, en particulier dans un passé récent, Philippe Dujardin, Olivier Frérot et Romy Sauvayre. Il remercie les chercheurs avec qui il a discuté ces questions, avec une mention spéciale pour les membres du comité scientifique des Ateliers sur la contradiction de Saint-Étienne (et parmi eux Jean-Louis Léonhardt pour son aide en matière bibliographique). Il pense à tous les auteurs dont les œuvres ont nourri sa réflexion et regrette de ne pouvoir les citer tous ni toutes (la citation ne signifie pas toujours une adhésion complète aux opinions des auteurs mentionnés). Il remercie les deux relecteurs anonymes, un francophone et un anglophone, pour leurs encouragements et leurs critiques utiles pour la révision du manuscrit.

\section{Bibliographie}

Alegria, Jésus

1983 Le développement de la notion d'espace et de temps, dans L'Espace et le temps, Paris : Le Seuil, 165-178.

Aristote

1961 Physique, Paris : Les Belles Lettres, traduction H. Carteron, texte bilingue, IV ${ }^{\text {e }}$ siècle av. J.C.

1997 Organon, I Catégories, II De l'interprétation, Vrin, traduction J. Tricot, IV ${ }^{\mathrm{e}}$ siècle av. J.C.

Asic, Tijana

2004 La représentation cognitive du temps et de l'espace : étude pragmatique des données linguistiques en français et dans d'autres langues, Thèse de doctorat, Université de Genève et Université de Lyon 2.

Assis, Andre K.T.

1999 Relational Mechanics, Montréal : Apeiron.

Barbour, Julian B.

1982 Relational concepts of space and time, British Journal for the Philosophy of Science, 33(3), 251-274.

Berthoz, Alain

1997 Le sens du mouvement, Paris : Odile Jacob. 
Bitbol, Michel

2010 De l'intérieur du monde. Pour une philosophie et une science des relations, Paris : Flammarion.

Chatelet, Gilles

1993 Les enjeux du mobile; mathématiques, physique, philosophie, Paris : Le Seuil.

Chen, X.

2005 Three dimensional time theory : To unify the principles of basic quantum physics and relativity. arXiv: quant-ph/0510010v1.

DAVID, PAScAL

2004 Présent, passé, futur, dans Vocabulaire européen des philosophies, édité par Cassin, Barbara, Le Seuil, 1016-1021.

Demers, P.

1975 Symétrisation de la longueur et du temps dans un espace de Lorentz $C^{3}$ en algèbre linéaire, pouvant servir en théorie trichromatique des couleurs, Can. J. Phys., 53, 1687-1688.

Descartes, René

1644 Euvres complètes, Bibliothèque de la Pléiade, Paris : Gallimard, chap. Les principes de la philosophie, 551-692.

Dujardin, Philippe

2009 De quoi sommes-nous contemporains? Essai d'anthropologie politique, Sens public, 11-12, 11-92.

Dujardin, Philippe \& Guy, Bernard

2011 Sur les conditions de la pensée de la relation. Échanges entre un anthropologue et un physicien, dans Actes des $2^{e}$ Ateliers sur la contradiction, Presses des Mines.

EARMAN, JOHN

1990 World enough and space-time; absolute versus relational theories of space and time, Cambridge : MIT Press.

Einstein, Albert

1905 Zur Elektrodynamik bewegter Körper, Annalen der Physik, 17, 891921, Sur l'électrodynamique des corps en mouvement.

Franco, J. A.

2006 Vectorial lorentz transformations, Electronic Journal of Theoretical Physics, 9, 35-64. 
Greenberg, Marvin Jay

1997 Euclidean and non-Euclidean geometries. Development and history, New York : W.H. Freeman Company.

Guy, Bernard

2004 L'éclair et le tonnerre, promenades entre l'espace et le temps (à propos de la théorie de la relativité), Paris : Éditions EPU.

2008 Particles, scale, time construction and the second law of thermodynamics, dans Meeting the entropy challenge, an international thermodynamics conference in honor and memory of Professor Joseph Henry Keenan, édité par Beretta, Ghonien \& Hatsopoulos, Mit, New York : The American Institute of Physics, 174-179.

2010a Contradictions dans la pensée de l'espace, du temps et du mouvement, dans Actes des ateliers sur la contradiction, Paris : Presses des Mines, 85-92.

2010b Groupes sociaux, espace, temps, dialogue entre un physicien et un anthropologue.

http://hal archives-ouvertes.fr/hal-00468407/en/.

2010c A modified law of gravitation taking account of the relative speeds of the moving masses. A preliminary study.

http://hal . archives-ouvertes.fr/hal-00472210/fr.

2010d Les relations de Lorentz et le temps : proposition d'utilisation d'un paramètre temporel tri-dimensionnel défini par un déplacement; la question du temps en physique.

www .archive.org/details/LesRelationsDeLorentz\

$\backslash$ EtLeTempspropositionDutilisationDun.

Hagège, Claude

2009 Dictionnaire amoureux des langues, Paris : Plon, Odile Jacob.

HÉRACLITE

1964 Fragments. Les penseurs grecs avant Socrate, de Thalès de Milet à Prodicos, Paris : Garnier-Flammarion, $\mathrm{VI}^{\mathrm{e}}$ siècle avant J.C., édité par Jean Voilquin.

IMBERT, MiCHEL

1983 L'espace, le temps et la neurobiologie, dans L'Espace et le temps, édité par NoËL, É., Paris : Le Seuil, 179-192.

Jullien, FrançOIS

1993 Figures de l'immanence, pour une lecture philosophique du Yiking, le « classique du changement », Paris : Grasset. 
2006 Si parler va sans dire, du logos et autres ressources, Paris : Le Seuil. Kant, Emmanuel

1781 Critique de la raison pure, Paris : Gallimard, 3 tomes, 1985.

LÉONHARDT, JEAN-Louis

2008 Le rationalisme est-il rationnel? L'homme de science et sa raison, Lyon : Parangon.

Mach, ERnst

1904 La mécanique, exposé historique et critique de son développement, Paris : Librairie scientifique A. Hermann.

Maldiney, Henry

2001 Existence, crise et création, Paris : Encre marine.

Mizony, Michel

2010 Sur le pluralisme théorique : de Kant à Poincaré; ou comment gérer les paradoxes en sciences, dans Ateliers sur la contradiction, édité par Guy, Bernard, Paris : Presses des Mines.

Montaigne, Michel Eyquem De

1580 Les Essais, Paris : Presses Universitaires de France, 1965.

MorAïs, José

1983 La perception de l'espace et du temps, dans $L^{\prime} E s p a c e$ et le temps, édité par NoËL, É., Paris : Le Seuil, 149-164.

MORIN, EDGAR

1986 La méthode. 3. La connaissance de la connaissance, Paris : Le Seuil.

2005 Introductionà la pensée complexe, Paris : Le Seuil.

NewTon, IsaAC

1687 Principes mathématiques de la philosophie naturelle, Jacques Gabay, traduction Madame la marquise du Chastelet, 2 volumes, ré-impression, 1990.

Ninio, JACQUeS

1983 La géométrie, l'œil et le cerveau, dans L'Espace et le temps, édité par NoËL, É., Paris : Le Seuil, 193-205.

Nodland, Borge \& Ralston, John P.

1997 Indication of anisotropy in electromagnetic propagation over cosmological distances, Phys. Rev. Lett., 78(16), 3043-3046. 
Pappas, Panos T.

1978 Physics in six dimensions : An axiomatic formulation, Lett. Nuovo Cimento, 22(15), 601-607.

1979 The "three-dimensional time" equation, Lett. Nuovo Cimento, 25(14), 429-434.

Pascal, Blaise

1670 Pensées, Paris : Le Seuil, 1962.

Piaget, Jean

1967 La construction du réel chez l'enfant, Neuchatel : Delachaux et Niestlé.

Poincaré, Henri

1902 La Science et l'Hypothèse, Paris : Flammarion, 1968.

1905 La Valeur de la science, Paris : Flammarion, 1970.

Pomian, Krzysztof

1984 L'Ordre du temps, Paris : Gallimard.

Riceeur, Paul

1969 Le Conflit des interprétations, essais d'herméneutique, Paris : Le Seuil.

RöSSleR, OtTo

1998 Endophysics, the world as an interface, Londres : World Scientific.

Rovelli, Carlo

1990 Quantum mechanics without time : A model, Physical Review, 42(8), 2638-2646.

1991 Time in quantum gravity : An hypothesis, Physical Review, 43(2), $442-456$.

Selleri, Franco

1998 Open questions in relativistic physics, Montréal : Apeiron.

SMOLin, LeE

2006 The trouble with physics. The rise of string theory, the fall of a science and what comes next, Boston : Houghton Mifflin Harcourt publishing company, traduction française par Alexeї Grinbaum Rien ne va plus en physique, l'échec de la théorie des cordes, Paris : Dunod, 2007. 
Teissier, Bernard

2009 Géométrie et cognition : l'exemple du continu, dans Ouvrir la logique au monde, édité par Joinet, J.-B. \& Tronçon, S., Paris : Hermann.

Tsabary, Guy \& Censor, Aviv

2004 An alternative mathematical model for special relativity. ArXiv:math-ph/0402054v119Feb2004.

WeBb, DAVID

2006 Heidegger et Weyl : nombre, mouvement et continuité, Noesis. Revue en ligne, 9, 85-102. http://noesis.revues.org/index275.html.

Whitehead, Alfred North

1922 The principle of relativity, with applications ot physical sciences, Cambridge : The University Press.

Zirno, G.

1979a On the theoretical reliability of a three-temporal Lorentz transformation, Lett. Nuovo Cimento, 24(6), 171-174.

1979b On the possibility of a three-temporal Lorentz transformation, Phys. Lett., $70 \mathrm{~A}(2), 77-78$. 